ARTICLE

\title{
Energy storing bricks for stationary PEDOT supercapacitors
}

Hongmin Wang (1) ${ }^{1}$, Yifan Diao ${ }^{2}$, Yang Lu ${ }^{2}$, Haoru Yang ${ }^{1}$, Qingjun Zhou ${ }^{2}$, Kenneth Chrulski (i) ${ }^{1} \&$ Julio M. D'Arcy (1) 1,2四

Fired brick is a universal building material, produced by thousand-year-old technology, that throughout history has seldom served any other purpose. Here, we develop a scalable, costeffective and versatile chemical synthesis using a fired brick to control oxidative radical polymerization and deposition of a nanofibrillar coating of the conducting polymer poly $(3,4-$ ethylenedioxythiophene) (PEDOT). A fired brick's open microstructure, mechanical robustness and $\sim 8 \mathrm{wt} \% \alpha-\mathrm{Fe}_{2} \mathrm{O}_{3}$ content afford an ideal substrate for developing electrochemical PEDOT electrodes and stationary supercapacitors that readily stack into modules. Fiveminute epoxy serves as a waterproof case enabling the operation of our supercapacitors while submerged underwater and a gel electrolyte extends cycling stability to 10,000 cycles with $\sim 90 \%$ capacitance retention.

\footnotetext{
${ }^{1}$ Department of Chemistry, Washington University in St. Louis, St. Louis, MI 63130, USA. ${ }^{2}$ Institute of Material Science \& Engineering, Washington

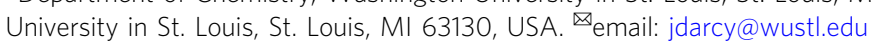


F red brick, typically used for construction and architectural esthetics, is one of the most durable materials with a 5000year history dating back to Neolithic China ${ }^{1}$. This masonry building block is commonly found in various red tones and mostly comprised of fused particles of silica $\left(\mathrm{SiO}_{2}\right)$, alumina $\left(\mathrm{Al}_{2} \mathrm{O}_{3}\right)$ and hematite $\left(\alpha-\mathrm{Fe}_{2} \mathrm{O}_{3}\right)^{2}$. The red color of a brick originates from hematite, a pigment first utilized by humans 73,000 years ago $^{3,4}$ and serving today as a low-cost naturally abundant inorganic precursor for catalysts ${ }^{5}$, magnets ${ }^{6}$, and alloys ${ }^{7}$. State-ofthe-art energy storage materials are also produced from hematite. For example, $\mathrm{FeN}_{x}, \mathrm{FeP}$, and $\mathrm{Li}_{5} \mathrm{FeO}_{4}$ are synthesized via anionic or cationic exchange for potassium-ion batteries, $\mathrm{Zn}$-air batteries, pseudocapacitors, and lithium-ion batteries ${ }^{8-11}$; electrochemical transformation of hematite leads to $\mathrm{FeOOH}$ supercapacitor anodes $^{12}$.

This work is inspired by our recently published rust-assisted vapor phase polymerization ${ }^{13}$. Chemistries enabled by hematite provide an opportunity for developing cutting-edge functionalities on a fired brick where $8 w \mathrm{wt} \% \quad \alpha-\mathrm{Fe}_{2} \mathrm{O}_{3}$ content and a 3D porous microstructure afford an ideal substrate for engineering a mechanically robust electrode. Here, we develop a supercapacitor using a brick's hematite microstructure as reactant to vapordeposit a nanofibrillar coating of the conducting polymer poly (3,4-ethylenedioxythiophene) (PEDOT). Vapor-phase synthesis leads to PEDOT coatings exhibiting a high electronic conductivity ${ }^{14}$ and facile charge transfer, making it an ideal route for producing electrodes ${ }^{15}$. This synthesis utilizes a brick's open microstructure and thermal stability to permeate acid and monomer vapor through its pores at $160{ }^{\circ} \mathrm{C}$ to control $\alpha-\mathrm{Fe}_{2} \mathrm{O}_{3}$ dissolution and $\mathrm{Fe}^{3+}$ hydrolysis with concomitant oxidative radical polymerization.

A symmetric brick-based supercapacitor shows an areal capacitance of $1.60 \mathrm{~F} \mathrm{~cm}^{-2}$ and energy density of $222 \mu \mathrm{Wh} \mathrm{cm} \mathrm{cm}^{-2}$ at a current density of $0.5 \mathrm{~mA} \mathrm{~cm}^{-2}$. This two-electrode-based measurement is collected using $1 \mathrm{M} \mathrm{H}_{2} \mathrm{SO}_{4}$ aqueous electrolyte under $1 \mathrm{~V}$ operating voltage window. To mimic a "brick-mortar-brick" structure, a supercapacitor is modified using a quasi-solid-state electrolyte (poly(vinyl alcohol)/ $1 \mathrm{M} \mathrm{H}_{2} \mathrm{SO}_{4}$ ) that also plays the role of binder and separator. Our devices are water-resistant because they are coated with an epoxy encapsulating layer that protects them enabling charge storage at temperatures between -20 and $60^{\circ} \mathrm{C}$. A supercapacitor is stable in ambient conditions undergoing 10,000 charge-discharge cycles with $\sim 100 \%$ coulombic efficiency and $\sim 90 \%$ capacitance retention. Moreover, a supercapacitor brick module is produced reaching a $3.6 \mathrm{~V}$ voltage window by connecting three devices in series.

\section{Results}

Conversion of a fired brick's $\alpha-\mathrm{Fe}_{2} \mathrm{O}_{3}$ to a PEDOT coating. Deposition of PEDOT nanofibers is initiated by dissolving $a-$ $\mathrm{Fe}_{2} \mathrm{O}_{3}$ at $160^{\circ} \mathrm{C}$ with $\mathrm{HCl}$ vapor; this process liberates $\mathrm{Fe}^{3+}$ ions, promotes hydrolysis and initiates precipitation of colloidal 1D FeOOH nuclei (Fig. 1a). As previously reported, partially dissolved $\mathrm{FeOOH}$ nuclei serving as templates oxidize 3,4-ethylenedioxythiophene (EDOT) monomer vapor and control oxidative radical polymerization ${ }^{13}$. Control of both stoichiometry and reaction time leads to PEDOT coatings with different thicknesses (Fig. 1b). We advance previous findings by demonstrating here that the synthesis utilizes two potential polymerization initiators, i.e., oxidant $\left(\mathrm{Fe}^{3+}\right)$ and acid $(\mathrm{HCl})$ where the former leads to oxidative radical polymerization and the latter, to acid-catalyzed polymerization (Supplementary Fig. 1). We readily control the polymerization mechanism as an acid-catalyzed polymerization typically produces nonconductive oligomers stemming from active chain termination ${ }^{16}$. Only PEDOT synthesized via oxidative radical polymerization exhibits long conjugation length, ordered chain packing, low electrical resistance, as well as high chemical and physical stability ${ }^{13,14,17,18}$.

In our polymerization mechanism, the acid concentration determines both dissolution rate and synthetic pathways. A high $\mathrm{H}^{+}$concentration facilitates the liberation of $\mathrm{Fe}^{3+}$ as well as oxidative radical polymerization while promoting acid-catalyzed polymerization that consumes EDOT and results in PEDOT of low conjugation length (Fig. 1c). To investigate the effect of $\mathrm{H}^{+}$ concentration, different volumes of concentrated $\mathrm{HCl}$ are added to the reactor and the $\mathrm{HCl}$ vapor concentration is calculated by dividing moles of $\mathrm{HCl}$ with reactor volume (assuming total evaporation). Using a $\mathrm{HCl}$ vapor concentration less than $4.8 \mathrm{mM}$ leads to an incomplete reaction because both oxidative radical polymerization and acid-catalyzed polymerization are impeded (Supplementary Fig. 2a). Increasing concentration to $14 \mathrm{mM}$ liberates $\mathrm{Fe}^{3+}$ and promotes oxidative radical polymerization resulting in PEDOT of low electrical resistance, whereas concentrations above $14 \mathrm{mM}$ activate the acid-catalyzed polymerization pathway resulting in uncontrolled reactions (Supplementary Fig. 2b). When oxidative radical polymerization dominates, the EDOT vapor concentration determines the thickness of a polymer coating and its electrical resistance (Supplementary Fig. 2a, c).

Synthesis starts when a brick and chemical reactants are heated together in a sealed vessel; chemical and physical changes on a brick are monitored by collecting sample aliquots at different time intervals. Notably, there is no color change on a brick's red surface during the first $4 \mathrm{~h}$ of reaction because brick dissolution is the rate-limiting step in our mixed synthetic mechanism that consists of (1) evaporation, (2) dissolution, (3) hydrolysis, and (4) polymerization. A blue PEDOT coating is visible on a brick $4 \mathrm{~h}$ after initiating a reaction and its thickness increases inversely proportional with electrical resistance until the end of reaction at $14 \mathrm{~h}$. An extended polymerization time increases the polymer coating's two-point probe electrical resistance (Supplementary Fig. 2d) because PEDOT loses dopant during heating ${ }^{18}$; fortunately, post-synthetic doping lowers the electrical resistance. Our synthesis produces a $400 \mu \mathrm{m}$ thick nanofibrillar PEDOT coating (2.8 wt\%) exhibiting $2 \Omega$ two-point probe electrical resistance and nanofibers characterized by a $\sim 30 \mu \mathrm{m}$ length and $\sim 190 \mathrm{~nm}$ diameter (Supplementary Fig. 3a-d). Polymer, purified by repeated rinses in methanol, is comprised of $\mathrm{S}, \mathrm{C}, \mathrm{O}$, and doped by $\mathrm{Cl}^{-}$in situ during polymerization as shown in energydispersive X-ray spectra (Supplementary Fig. 3e).

Our synthesis is general and applicable to different types of bricks. Here, three types of bricks (type 1-3) with different gravel $\left(\mathrm{SiO}_{2}\right)$ sizes and porosities are investigated (Fig. 2). Type 1 brick shows the most open microstructure (Fig. 2a) that facilitates reagent vapor diffusion, therefore utilized for the synthesis study in Fig. 1 and Supplementary Figs. 1-3. Powder X-ray diffraction of pulverized type 1 brick shows that $\mathrm{SiO}_{2}$ is the major phase while $\alpha-\mathrm{Fe}_{2} \mathrm{O}_{3}$ and $\mathrm{Al}_{2} \mathrm{O}_{3}$ are minor phases (Fig. 2c). These three types of bricks possess similar inorganic composition and concentration as shown by powder X-ray diffraction patterns (Fig. 2d). Porosity differences between brick types stem from various gravel sizes and manufacturing variables such as water content before sintering, sintering temperature and duration. Vapor-phase synthesis produces a contiguous polymer coating over the entire brick surface because dissolution generates an aqueous $\mathrm{Fe}^{3+}$ layer that coats inert gravel sites. Under identical reaction stoichiometries and time, PEDOT nanofibers of high aspect-ratio coat all types of bricks homogeneously (Fig. 2b). Our polymer coating technology is scalable (Fig. 2e) and patternable (Supplementary Fig. 4a) as demonstrated using type 1 brick. Nanofibrillar PEDOT coatings on all types of bricks show linear 


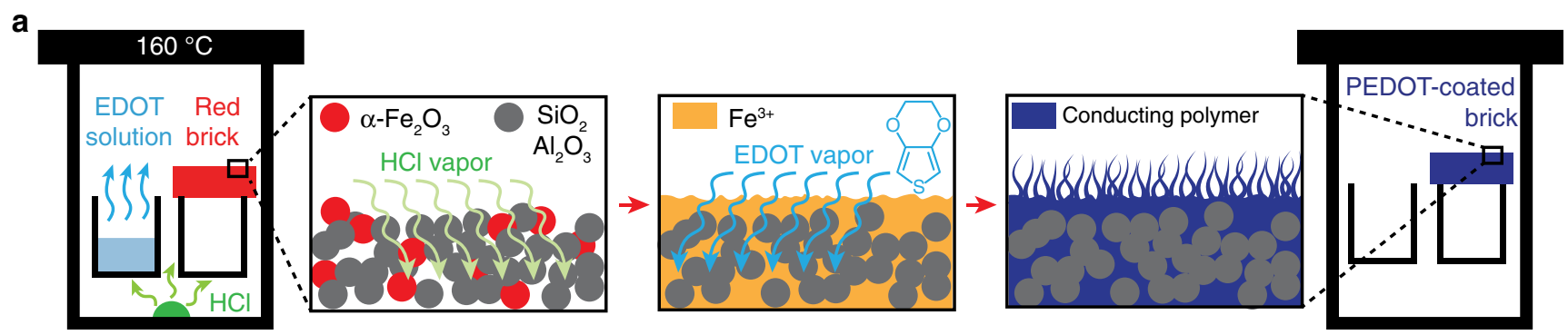

b

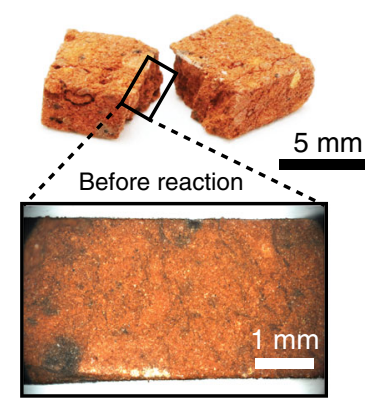

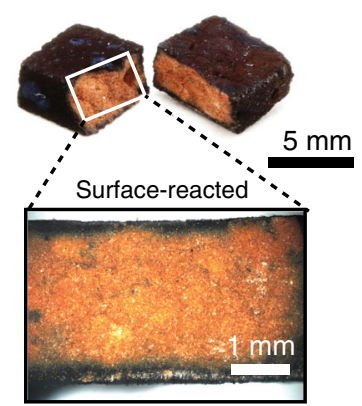

C

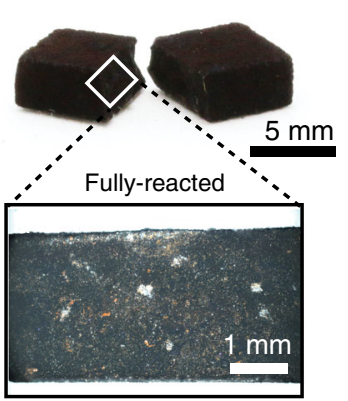

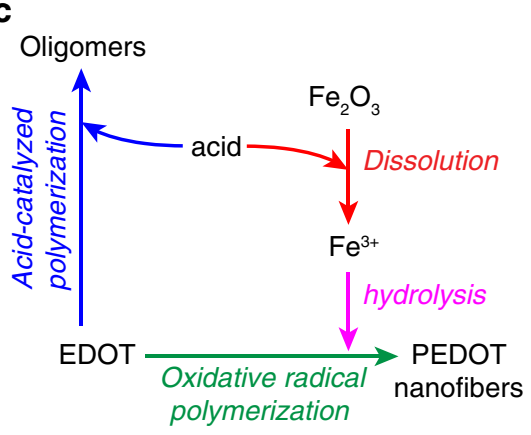

Fig. 1 Deposition of a nanofibrillar PEDOT coating on brick. a In a one-step reaction, a brick's $\alpha$ - $\mathrm{Fe}_{2} \mathrm{O}_{3}$ microstructure is partially dissolved by acid vapor to liberate $\mathrm{Fe}^{3+}$, promote hydrolysis and precipitation of $\mathrm{FeOOH}$ spindles that control oxidative radical polymerization. As previously reported, monomer vapor reacts with partially dissolved FeOOH nuclei resulting in preferential directional growth of high aspect ratio PEDOT nanofibers ${ }^{13}$. $\mathbf{b}$ The thickness of a PEDOT coating is controlled by reaction time and stoichiometry enabling a reaction to generate surface-polymerized PEDOT-coated bricks (core/shell architecture) or fully polymerized bricks (monolithic PEDOT architecture). c A reaction diagram shows the competition between acid-catalyzed polymerization and oxidative radical polymerization mechanisms present in our reactions.

current-voltage curves with slopes that indicate ohmic behaviors and similar electrical resistances $(\sim 7 \Omega)$ (Fig. 3a). Note that a twopoint probe multimeter measurement leads to a lower value $(2 \Omega)$ because of a wide tip diameter that lowers contact resistance.

In order to study the effect of polymer coatings on the porosity of bricks, water absorption experiments are carried out before and after synthesis. A PEDOT-coated type 1 brick absorbs the most weight of water among the three types of polymer-coated bricks due to its open pore structure and large pore size and volume (Fig. 2a). A nanofibrillar PEDOT coating on type 1 brick exhibits minimal delamination via Scotch tape tests whereas a coating of the commercial product PEDOT:poly(styrenesulfonate) peels off readily and completely. Note that both type 2 and type 3 bricks show partial delamination (Fig. 3c) of our polymer coating because of a semi-closed brick microstructure (Fig. 2a) that impedes vapor reactant diffusion (Fig. 3e, f). Cross-sectional scanning electron micrographs reveal poor PEDOT interpenetration into the brick's pores, this leads to a surface localized coating with minimal anchoring that is prone to delamination. Fortunately, type 1 brick's open microstructure enables reagent vapor diffusion producing in situ PEDOT grafting; a nanofibrillar polymer coating is embedded as a network throughout pores resulting in strong adhesion (Fig. 3d). The addition of $\alpha-\mathrm{Fe}_{2} \mathrm{O}_{3}$ particles enables deposition of PEDOT coatings on customized substrates such as concrete road pavers (Supplementary Fig. 4b) and Portland-based white concrete (Supplementary Fig. 4c).

Nanofibrillar PEDOT-coated brick electrochemical electrode. We present a summary of geometries and mass loadings for electrodes and devices in Supplementary Table 1 while areal, gravimetric, and volumetric capacitances, energy and power densities are shown in Supplementary Table 2 (calculations in detail are included in "Supplementary Methods" section). We choose areal metrics for evaluating our electrodes and devices because area is a practical parameter for evaluating our wall-like brick-based supercapacitor and it is directly related to the thin polymer coating (Supplementary Fig. 5) present on a wall. Areal metrics facilitate the estimation of capacitance and energy density delivered by a brick wall (Supplementary Discussion).

A $1 \mathrm{~cm} \times 0.5 \mathrm{~cm} \times 0.28 \mathrm{~cm}$ nanofibrillar PEDOT-coated type 1 brick $\left(0.14 \mathrm{~cm}^{3}\right)$ weighing $249 \mathrm{mg}$ and carrying $6.97 \mathrm{mg}$ PEDOT is fabricated into an electrode with one $1 \mathrm{~cm} \times 0.5 \mathrm{~cm}$ face exposed (Fig. 4a). When calculating areal metrics, we use the area of the face exposed to electrolyte $\left(0.5 \mathrm{~cm}^{2}\right)$. The electrode exhibits a quasi-rectangular shaped three-electrode cyclic voltammogram and a capacitance of $2.61 \mathrm{~F} \mathrm{~cm}^{-2}\left(187 \mathrm{~F} \mathrm{~g}^{-1}\right.$ based on PEDOT's mass) at $2 \mathrm{mV} \mathrm{s}^{-1}$ in $1 \mathrm{M} \mathrm{H}_{2} \mathrm{SO}_{4}$ (Fig. 4b). The low scan rate of $2 \mathrm{mV} \mathrm{s}^{-1}$ enables enough time for charge transfer in the thick electrode to calculate maximum capacitance and energy density. We summarize capacitances obtained at scan rates from 2 to $100 \mathrm{mV} \mathrm{s}^{-1}$ in Supplementary Fig. 6a. The $\mathrm{Fe}^{3+} / \mathrm{Fe}^{2+}$ redox pair at $0.37 \mathrm{~V}$ and $0.49 \mathrm{~V}$ (vs. $\mathrm{Ag} / \mathrm{AgCl}$ ) arise due to iron species in brick and disappears as scan rate increases to $25 \mathrm{mV} \mathrm{s}^{-1}$ because Faradaic processes in PEDOT are faster than those occurring from solid $\mathrm{Fe}_{2} \mathrm{O}_{3}$ (Fig. 4c inset) ${ }^{15,19}$. Rate performance tests demonstrate capacitive behavior as scan rate increases to $100 \mathrm{mV} \mathrm{s}^{-1}$; however, due to limited charge transport the curve changes to fusiform shape ${ }^{20}$ (Fig. 4c). Capacitance is also dependent on the aqueous electrolyte and sulfuric acid leads to greater capacitance $\left(1.64 \mathrm{~F} \mathrm{~cm}^{-2}\right)$ than sodium sulfate $(0.878 \mathrm{~F}$ $\mathrm{cm}^{-2}$ ) at $25 \mathrm{mV} \mathrm{s}^{-1}$ (Fig. $4 \mathrm{~d}$ ). This drastic difference is plausibly due to higher ionic mobility in $\mathrm{H}^{+}\left(36.23 \times 10^{-8} \mathrm{~m}^{2} \mathrm{~V}^{-1} \mathrm{~s}^{-1}\right)$ vs. $\mathrm{Na}^{+}\left(5.19 \times 10^{-8} \mathrm{~m}^{2} \mathrm{~V}^{-1} \mathrm{~s}^{-1}\right)$ or a lower electrical resistance caused by doping at low $\mathrm{pH}^{14}$. We probed this behavior further using electrochemical impedance spectroscopy. Nyquist plots and an equivalent circuit diagram demonstrate a significantly lower ion diffusion resistance for $\mathrm{H}_{2} \mathrm{SO}_{4}(1.7 \Omega)$ vs. $\mathrm{Na}_{2} \mathrm{SO}_{4}(4.6 \Omega)$ and minimal change in electrode material electrical resistance at low $\mathrm{pH}$ (Fig. 4e). To test the effect of an acidic electrolyte on a brick's inorganic species, we pulverize and homogenize the entire 
a

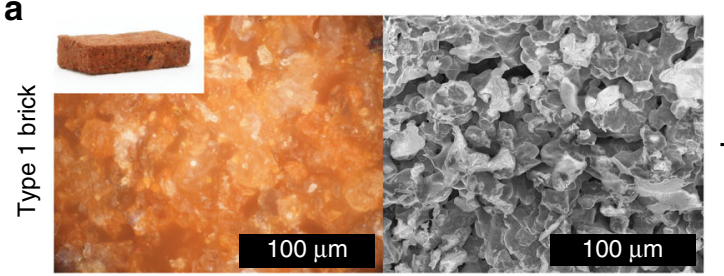

b
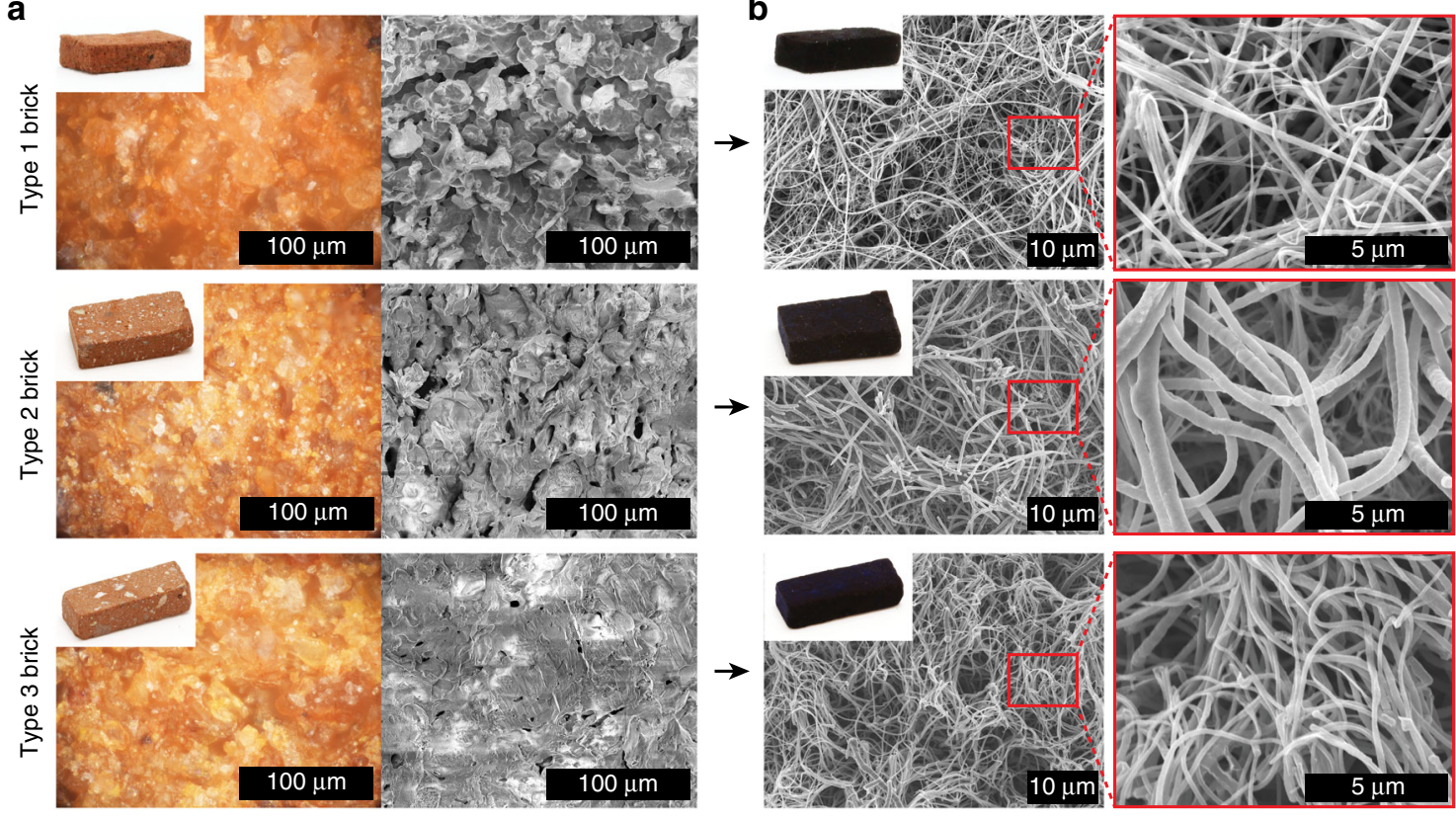

C

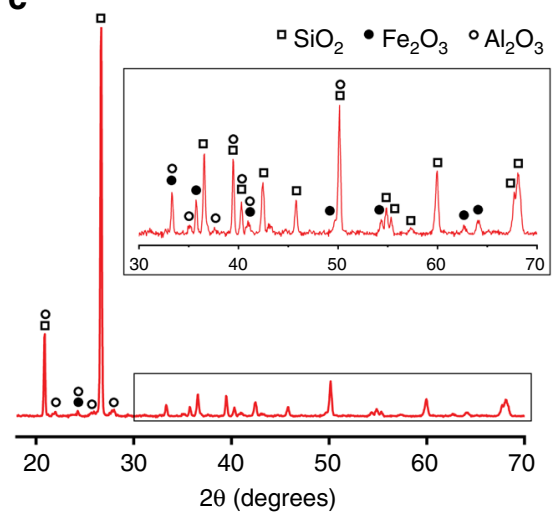

d

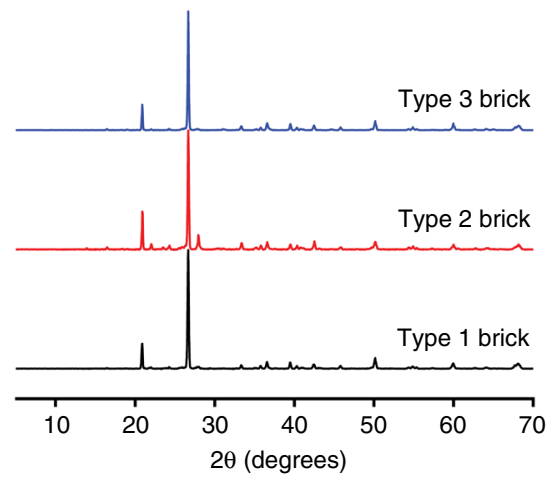

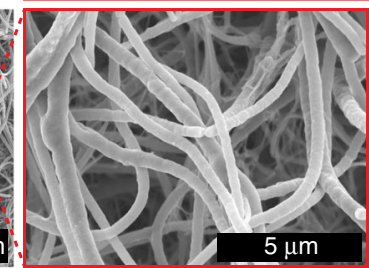

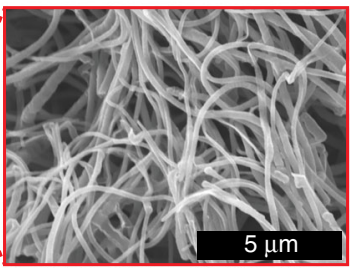

e

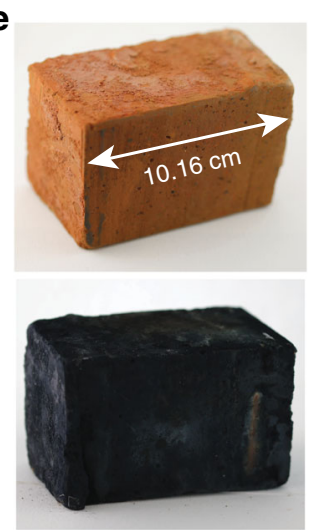

Fig. 2 Nanofibrillar PEDOT-coatings on different types of bricks. a Three types of red fired bricks are utilized for synthesis. All types show homogeneous red-orange color under the optical microscope, indicating uniform $\alpha-\mathrm{Fe}_{2} \mathrm{O}_{3}$ distribution. White spots on bricks (left top inset) show the gravel ( $\mathrm{SiO} \mathrm{O}_{2}$ ) sizes increasing from type 1 to type 3 brick. Scanning electron micrographs exhibit different porosity for these three types of bricks with pore sizes decreasing from type 1 to type 3. b After synthesis, all types of bricks are homogeneously coated by PEDOT nanofibers of similar aspect ratio. $\mathbf{c}$ Powder $\mathrm{X}$-ray diffraction pattern for type 1 brick shows a composition comprised of $\mathrm{SiO}_{2}, \alpha-\mathrm{Fe}_{2} \mathrm{O}_{3}$, and $\mathrm{Al}_{2} \mathrm{O}_{3}$. d Type 2 and type 3 bricks show similar patterns and crystalline components. The sharper and stronger peak at $2 \theta=28^{\circ}$ from type 2 brick is due to its higher $\mathrm{Al}_{2} \mathrm{O}_{3}$ content or crystallinity. e Synthesis is scalable to decimeter-sized bricks.

polymer-coated brick electrode using mortar and pestle. Powder $\mathrm{X}$-ray diffraction is carried out before and after electrodes are cycled in $1 \mathrm{M} \mathrm{H}_{2} \mathrm{SO}_{4}$ using sequential scan rates of 2, 5, 10, 25, 50, and $100 \mathrm{mV} \mathrm{s}^{-1}$ (10 cycles each). Diffraction patterns remain unchanged demonstrating that most inorganic species in a brick remain unaffected by synthesis and electrochemical cycling (Fig. 4f).

To quantify dissolution of a brick's $\alpha-\mathrm{Fe}_{2} \mathrm{O}_{3}$ and $\mathrm{Al}_{2} \mathrm{O}_{3}$ by $1 \mathrm{M}$ $\mathrm{H}_{2} \mathrm{SO}_{4}$, we perform inductive coupled plasma mass spectrometry on the electrolyte after cycling experiments (Supplementary Fig. $6 \mathrm{~b}, \mathrm{c})$. For a precise calculation, we control electrolyte volume $(5 \mathrm{~mL})$ and electrode mass $(249 \mathrm{mg})$. Analysis shows negligible concentrations of $\mathrm{Fe}\left(4.44 \mu \mathrm{g} \mathrm{mL}^{-1}\right)$ and $\mathrm{Al}\left(1.97 \mu \mathrm{g} \mathrm{mL}^{-1}\right)$ in the electrolyte after cycling, these concentrations are equivalent to mass losses (based on the entire brick) of 0.0127 and $0.0075 \mathrm{wt} \%$ for $\alpha-\mathrm{Fe}_{2} \mathrm{O}_{3}$ and $\mathrm{Al}_{2} \mathrm{O}_{3}$, respectively. These results confirm that inorganic species in a brick are preserved after electrochemical cycling.
Symmetric nanofibrillar PEDOT-coated brick supercapacitor. Two nanofibrillar PEDOT-coated bricks $(1 \mathrm{~cm} \times 0.5 \mathrm{~cm} \times$ $0.28 \mathrm{~cm}$ ) serve as electrodes in a symmetric supercapacitor $(1 \mathrm{~cm} \times 0.5 \mathrm{~cm} \times 0.5625 \mathrm{~cm})$ using $1 \mathrm{M} \mathrm{H}_{2} \mathrm{SO}_{4}$ aqueous electrolyte (Fig. 5a upper row and Supplementary Fig. 7a). A device consists of a volume $\left(0.28 \mathrm{~cm}^{3}\right)$ encompassing two PEDOT-coated bricks and a separator and its total mass $(499 \mathrm{mg})$ includes $13.94 \mathrm{mg}$ of PEDOT. Nyquist plot shows an aggregated internal resistance of $3 \Omega$ and a line with a $\sim 5^{\circ}$ slope between semicircle and lowfrequency domain (Warburg region) (Supplementary Fig. 7b). This line is characteristic of thick electrodes where a tortuous path stifles ion diffusion. At the low-frequency region, the curve tends to form an arc because of Nernst diffusion impedance of $\mathrm{H}^{+}$and $\mathrm{SO}_{4}{ }^{2-}$ in the electrolyte ${ }^{21}$; this plausibly stems from an increased ion diffusion distance caused by a separation gap between anode and cathode. Cyclic voltammogram shows a quasi-rectangular shape between 0 and $1 \mathrm{~V}$ (collected at $2 \mathrm{mV} \mathrm{s}^{-1}$ ) leading to a device areal capacitance of $1.59 \mathrm{~F} \mathrm{~cm}^{-2}$ 
a

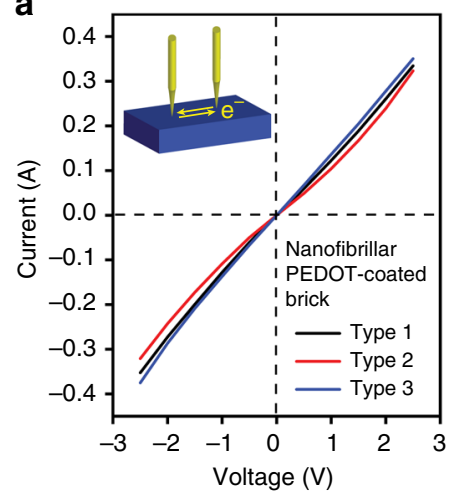

b

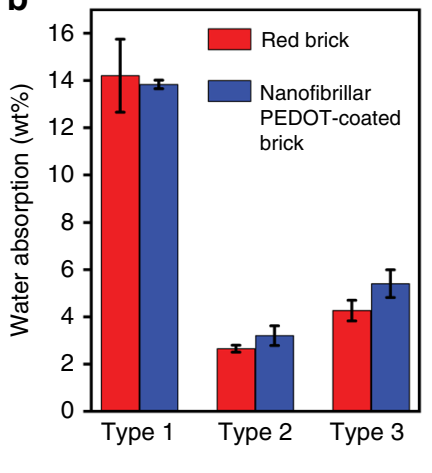

C

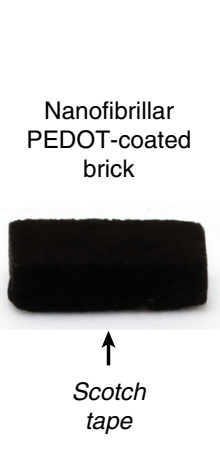

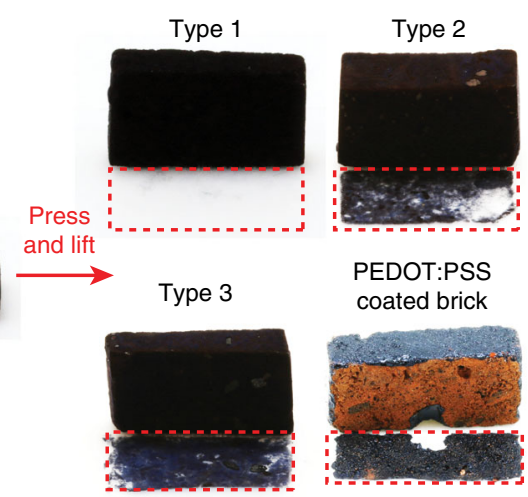

\section{d}
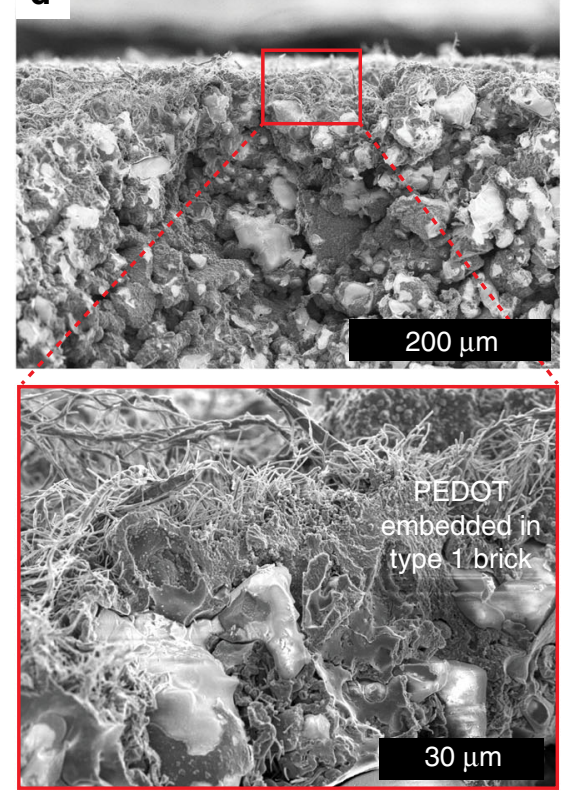
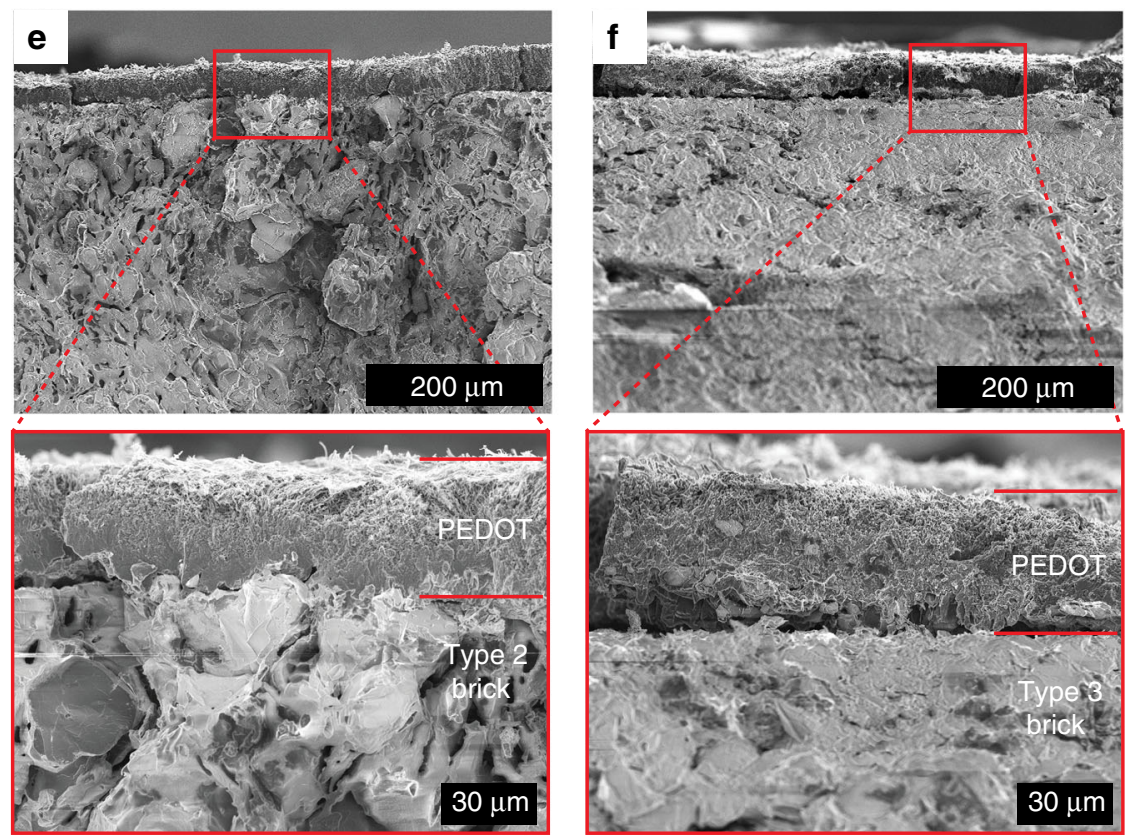

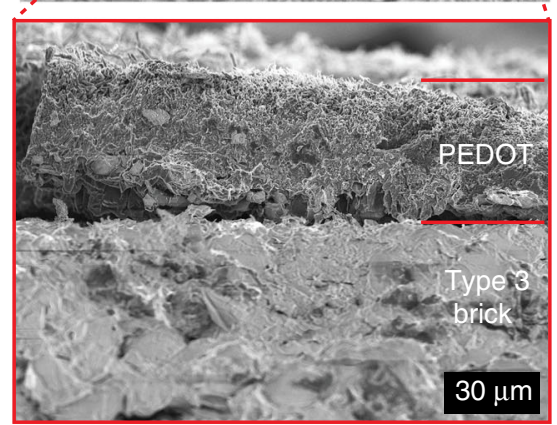

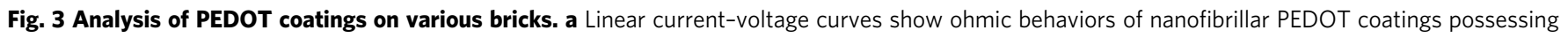

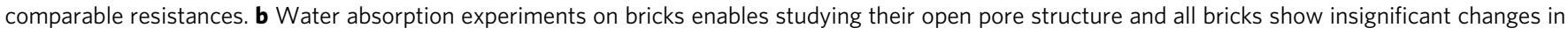
water absorption after PEDOT coatings with type 1 brick absorbing the most water due to a higher porosity. c A nanofibrillar PEDOT coating exhibits superior adhesion on type 1 brick versus other types during Scotch tape tests. Unfortunately, the commercial product PEDOT:poly(styrene sulfonate) delaminates completely after coated on type 1 brick. d Cross-sectional scanning electron micrographs show the embedded PEDOT network in type 1 brick leading to robust PEDOT adhesion. Type 2 (e) and type 3 (f) bricks show distinct boundaries between PEDOT and brick that are prone to delamination.

calculated using the electrode area directly in contact with separator $\left(0.5 \mathrm{~cm}^{2}\right)$ (Fig. 5b, black curve and Supplementary Fig. 7c). In our device, a nanofibrillar PEDOT coating covers all six faces of a brick, two of the larger faces $(1 \mathrm{~cm} \times 0.5 \mathrm{~cm})$ are directed towards each other and during cycling, ions travel through the inner brick pores resulting in an electrochemical contribution by the other faces. The areal metrics of this aqueous electrolyte supercapacitor are thus qualitative.

Our supercapacitor possesses low internal resistance resulting in a low IR drop $(0.01 \mathrm{~V})$ during galvanostatic charge-discharge experiments at $0.5 \mathrm{~mA} \mathrm{~cm} \mathrm{c}^{-2}$ current density in a $1 \mathrm{~V}$ window (Supplementary Fig. 7d). These curves demonstrate a device areal capacitance of $1.60 \mathrm{~F} \mathrm{~cm}^{-2}\left(2.84 \mathrm{~F} \mathrm{~cm}^{-3}\right.$ for volumetric) as well as areal energy and power densities of 222 and $0.25 \mathrm{~mW} \mathrm{~cm}^{-2}$, respectively (394 $\mu \mathrm{Wh} \mathrm{cm} \mathrm{cm}^{-3}$ and $0.44 \mathrm{~mW} \mathrm{~cm}^{-3}$ for volumetric). High power density $\left(12.5 \mathrm{~mW} \mathrm{~cm}^{-2}\right)$ is obtained at a current density of $25 \mathrm{~mA} \mathrm{~cm}^{-2}$ albeit with lowered capacitance $(0.706 \mathrm{~F}$ $\left.\mathrm{cm}^{-2}\right)$ and energy density $\left(98 \mu \mathrm{Wh} \mathrm{cm}^{-2}\right)$ because ion transport in our thick electrode is limited (shown by high IR drop of $0.4 \mathrm{~V}$ ). Our device works in an extended voltage window
$(1.2 \mathrm{~V})$ resulting in cyclic voltammograms and galvanostatic charge-discharge curves that retain shape (Supplementary Fig. 7e, f). However, the increased IR drop $(0.07 \mathrm{~V})$ at $1.2 \mathrm{~V}$ decreases coulombic efficiency as well as long term cycling stability leading to lower capacitance versus $1 \mathrm{~V}$ (Supplementary Fig. $7 \mathrm{~g}, \mathrm{~h}$ ). The energy densities at $1.2 \mathrm{~V}$ are higher than at $1 \mathrm{~V}$ as shown in a Ragone plot (Supplementary Fig. 7i) because a wider voltage window allows a device to store more charge (Supplementary Eq. 7 from "Supplementary Methods" section). Connecting three devices in series increases the voltage window to $3.6 \mathrm{~V}$, this also triples the internal resistance and reduces output current to onethird (Supplementary Fig. 8a). A tandem device reaches an output voltage of $2.685 \mathrm{~V}$ (charged at $4.5 \mathrm{~V}$ for $15 \mathrm{~s}$ ) and lights a white light-emitting diode for $11 \mathrm{~min}$. The output voltage decreases to the same level as the light-emitting diode's turn-on voltage $(2.546$ V) after discharging for $214 \mathrm{~s}$ (Supplementary Fig. $8 \mathrm{~b}-\mathrm{d}$ and Supplementary Movie 1).

Quasi-solid-state PEDOT-coated brick supercapacitor. To minimize electrolyte leakage, we develop a symmetric supercapacitor 
a
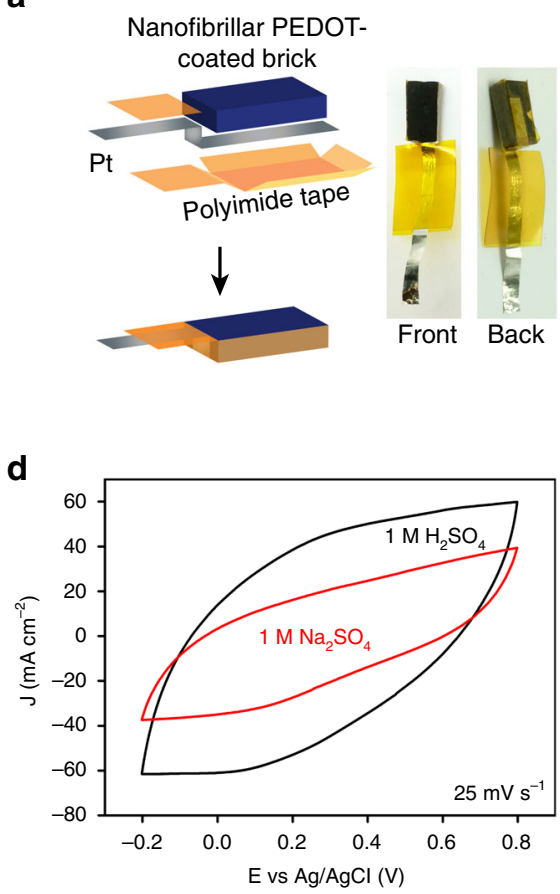

b

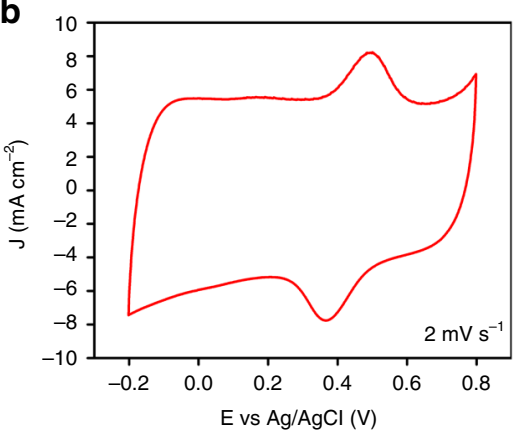

e

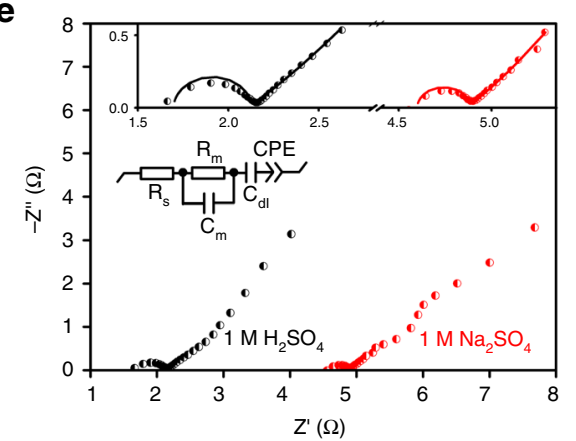

C

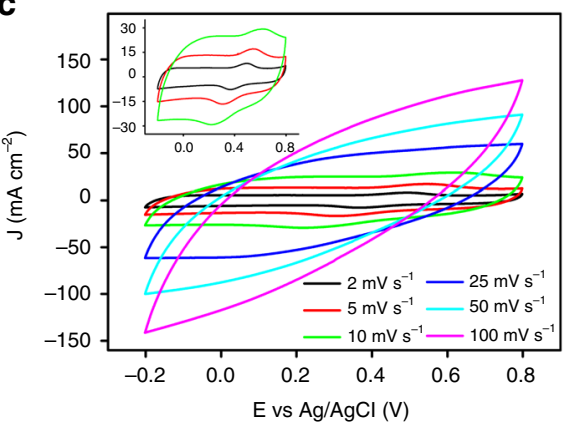

f

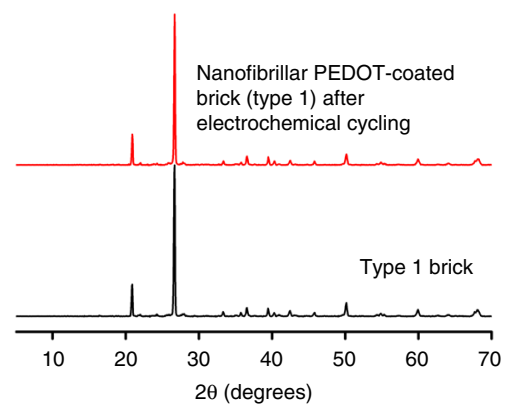

Fig. 4 Three-electrode characterization of nanofibrillar PEDOT-coated bricks. a Nanofibrillar PEDOT-coated type $1 \mathrm{brick}(1 \mathrm{~cm} \times 0.5 \mathrm{~cm} \times 0.28 \mathrm{~cm})$ is connected to a Pt current lead using polyimide tape that exposes a $1 \mathrm{~cm} \times 0.5 \mathrm{~cm}$ face to the electrolyte. $\mathbf{b}$ Cyclic voltammogram at $2 \mathrm{mV} \mathrm{s} \mathrm{s}^{-1}$ shows a quasirectangular shape stemming from PEDOT's capacitive behavior with $\mathrm{Fe}^{3+} / \mathrm{Fe}^{2+}$ redox pair peaks at 0.37 and $0.49 \mathrm{~V}\left(\mathrm{vs} . \mathrm{Ag} / \mathrm{AgCl}\right.$ ). c The $\mathrm{Fe}{ }^{3+} / \mathrm{Fe}^{2+}$ redox peaks disappear when scan rate increases to $25 \mathrm{mV} \mathrm{s}^{-1}$. d The electrode shows a smaller curve area for $1 \mathrm{M} \mathrm{Na}_{2} \mathrm{SO}_{4}$ electrolyte compared to $1 \mathrm{M} \mathrm{H}_{2} \mathrm{SO}_{4}$, indicating lower capacitance. e Nyquist plots collected using different electrolytes (inset shows equivalent circuit diagram) show fitted (solid lines) versus experimental data (segregated points). f Powder $\mathrm{X}$-ray diffraction shows identical patterns for pristine type 1 brick vs. PEDOT-coated type 1 brick after cycling in $1 \mathrm{M} \mathrm{H}_{2} \mathrm{SO}_{4}$ between -0.2 and $0.8 \mathrm{~V}$ (vs. $\mathrm{Ag} / \mathrm{AgCl}$ ) at scan rates of $2,5,10,25,50$, and $100 \mathrm{mV} \mathrm{s}^{-1}$ (10 cycles each).

$(1 \mathrm{~cm} \times 0.5 \mathrm{~cm} \times 0.63 \mathrm{~cm})$ using a poly $($ vinyl alcohol $) / 1 \mathrm{M} \mathrm{H}_{2} \mathrm{SO}_{4}$ gel that binds PEDOT-coated bricks and serves as electrolyte and separator (Fig. 5a lower row and Supplementary Fig. 9a). The gel electrolyte layer $(0.7 \mathrm{~mm}$ thick $)$ prevents bricks $(1 \mathrm{~cm} \times 0.5 \mathrm{~cm} \times 0.28 \mathrm{~cm})$ from short-circuiting and leads to enhanced adhesion between electrodes. This sandwich-type supercapacitor is $0.32 \mathrm{~cm}^{3}$ in volume, $518 \mathrm{mg}$ in weight (includes mass of electrodes and electrolyte) containing $13.94 \mathrm{mg}$ of PEDOT. In a tensile test, our electrode-gel-electrode structure withstands a shearing force equal to 1000 times the device's weight ${ }^{20}$ (Supplementary Fig. 9b). Intimate contact between gel and PEDOT nanofibers enhances charge transfer resulting in low internal resistance $(2.5 \Omega)$ and a linear Nyquist plot (Supplementary Fig. 9c). Device areal capacitance $\left(0.868 \mathrm{~F} \mathrm{~cm}^{-2}\right)$ and areal energy density $\left(121 \mu \mathrm{Wh} \mathrm{cm}^{-2}\right)$ originating from PEDOT-coated brick electrodes are calculated from galvanostatic charge-discharge curves $\left(1.38 \mathrm{~F} \mathrm{~cm}^{-3}\right.$ and $192 \mu \mathrm{Wh} \mathrm{cm}^{-3}$ for volumetric, collected at $\left.0.5 \mathrm{~mA} \mathrm{~cm}{ }^{-2}\right)$; cyclic voltammograms also show capacitive behavior (Fig. 5b, c, Supplementary Fig. 9d-g). The absence of redox peak from cyclic voltammogram indicates a minimal contribution from the $\alpha-\mathrm{Fe}_{2} \mathrm{O}_{3}$ present in a brick to energy storage. A gel electrolyte leads to $50 \%$ lower areal capacitance and energy density than an aqueous electrolyte because of stifled gel permeation kinetics throughout the electrode preventing access of ions to all non-sandwiched PEDOT-coated faces (Fig. 5a, Supplementary Fig. 9h).

Outdoor exposure is inevitable for a stationary supercapacitor and epoxy encapsulation affords a cost-effective, mechanically robust and waterproof housing. An epoxy-coated supercapacitor retains $\sim 90 \%$ of original capacitance and exhibits $\sim 100 \%$ coulombic efficiency after 10,000 charge-discharge cycles (collected at $25 \mathrm{~mA} \mathrm{~cm}^{-2}$ ) (Fig. 5d, red curves). This 5-min epoxy coating prevents water evaporation from the gel's hydrated ionic percolation network ${ }^{22}$ (Supplementary Fig. 10a) enabling 10,000 charge-discharge cycles at $5 \mathrm{~mA} \mathrm{~cm}^{-2}$ (640 h of continuous operation) with $\sim 87 \%$ capacitance retention (Fig. 5d, black curves). Gel electrolyte and encapsulation enables operation at temperatures between -20 and $60^{\circ} \mathrm{C}$ (this range covers most outdoor temperatures) as shown by cyclic voltammograms and Nyquist plots (Supplementary Fig. 10b, c). The capacitance increases proportionally with temperature due to enhanced ionic transport ${ }^{22}$; PEDOT remains capacitive after repeated reversible heating-cooling cycles (Supplementary Fig. 10d). Temperatures below $-20^{\circ} \mathrm{C}$ or above $60^{\circ} \mathrm{C}$ cause significant freezing or evaporation of water from the gel electrolyte leading to unstable electrical performance and breakage of epoxy seal.

Epoxy renders a stationary supercapacitor module waterproof. Three quasi-solid-state supercapacitors are sealed in epoxy pucks (Supplementary Fig. 9a) and connected in series by electrical wires. This entire module (including connection points) is coated by another layer of epoxy that leaves only cathode and anode exposed. After epoxy curing, the entire module is immersed in water except for two electrode-leads and is electrochemically cycled (electrical circuit shown in Fig. 5e inset and Supplementary Fig. 10e). The module's cyclic voltammograms (Fig. 5e) collected while immersed demonstrate stable behavior identical to preimmersion tests. A device charges to $3 \mathrm{~V}$ in $10 \mathrm{~s}$ while immersed in water and lights up a green light-emitting diode $(2.155 \mathrm{~V}$ forward voltage) for $\sim 10 \mathrm{~min}$ (Supplementary Fig. 10e, f). A gel electrolyte and our deposition technology enable scale up as demonstrated by connecting six large nanofibrillar PEDOTcoated brick electrodes $(2 \mathrm{~cm} \times 1 \mathrm{~cm} \times 1 \mathrm{~cm})$ in series resulting in a supercapacitor module that charges to $3 \mathrm{~V}$ in $5 \mathrm{~s}$ readily lighting up a green light-emitting diode (Fig. 5f, Supplementary Fig. 11). 
a

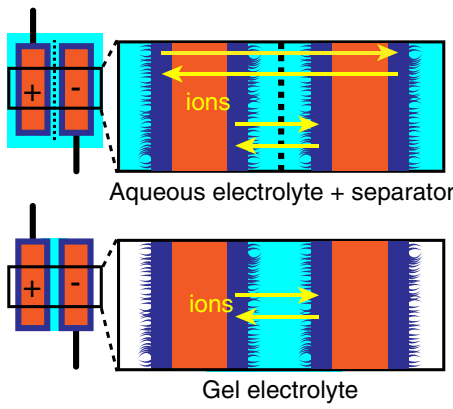

d

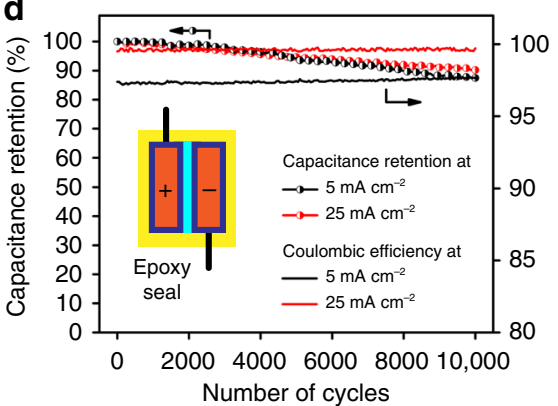

b
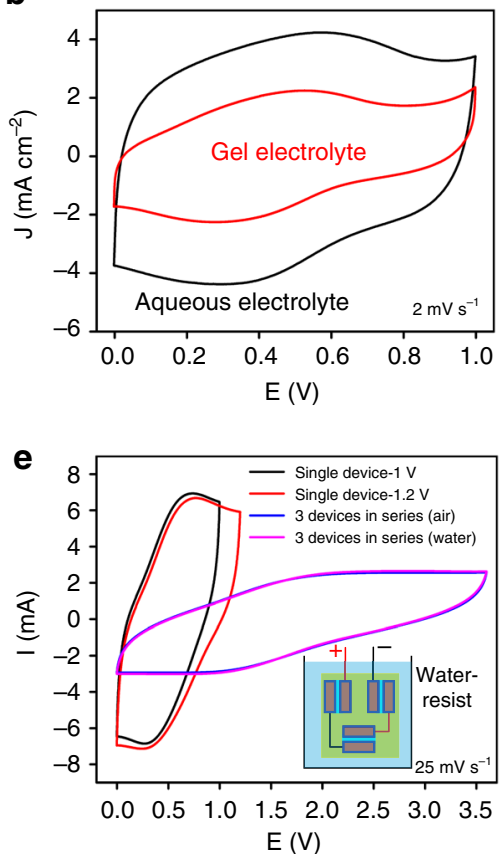

C
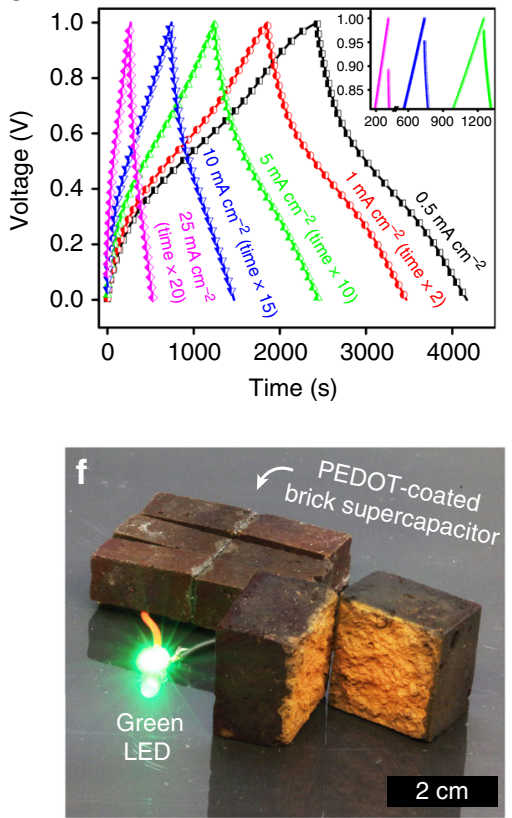

Fig. 5 Nanofibrillar PEDOT-coated bricks for supercapacitors. a Schematic illustration of aqueous electrolyte supercapacitor and quasi-solid-state supercapacitor shows different charge storage sites. The black dash line in the aqueous electrolyte device represents a separator. $\mathbf{b}$ Cyclic voltammograms for symmetric supercapacitors using $1 \mathrm{M} \mathrm{H}_{2} \mathrm{SO}_{4}$ aqueous electrolyte and poly(vinyl alcohol) $/ \mathrm{H}_{2} \mathrm{SO}_{4}$ gel electrolyte. c Galvanostatic charge-discharge profiles for quasi-solid-state device at current densities ranging between 0.5 and $25 \mathrm{~mA} \mathrm{~cm}^{-2}$; curves at 1, 5, 10, and $25 \mathrm{~mA} \mathrm{~cm}^{-2}$ are horizontally expanded 2x, 10x, 15x, and 20x, respectively. Inset shows IR drop at current densities of 5, 10, and $25 \mathrm{~mA} \mathrm{~cm}^{-2}$. d Quasi-solid-state supercapacitor charge-discharge curves after 10,000 cycles at 5 and $25 \mathrm{~mA} \mathrm{~cm}-2$ exhibit $87 \%$ and $90 \%$ capacitance retention, respectively (coulombic efficiency is 100\%). e Cyclic voltammograms for a single quasi-solid-state supercapacitor are collected at voltage windows of 1 and $1.2 \mathrm{~V}$; a tandem device (comprised of three supercapacitors connected in series) withstands a $3.6 \mathrm{~V}$ window. The tandem device is waterproof after coated by epoxy and exhibits a stable cyclic voltammogram. f Photograph shows a supercapacitor module lighting up a green light-emitting diode. This tandem device $(4 \mathrm{~cm} \times 3 \mathrm{~cm} \times 1 \mathrm{~cm})$ contains three supercapacitors $(4 \mathrm{~cm} \times 1 \mathrm{~cm} \times 1 \mathrm{~cm})$ connected in series; the core-shell structure of an electrode is also shown.

Notably, a brick wall constructed using our nanofibrillar PEDOTcoated bricks holds the potential to deliver a maximum device capacitance of $11.5 \mathrm{kF} \mathrm{m}^{-2}$ and an energy density of $1.61 \mathrm{Wh} \mathrm{m}^{-2}$ (Supplementary Fig. 12; Supplementary Discussion).

\section{Discussion}

This proof-of-concept work demonstrates how to store energy on the surface of a common brick using $a-\mathrm{Fe}_{2} \mathrm{O}_{3}$ as an oxidant precursor to control oxidative radical polymerization and conformally deposit a capacitive nanofibrillar PEDOT coating from the vapor phase. A brick's structural stability and open microstructure result in mechanically robust PEDOT-coated brick electrodes, that when connected in series and coated with epoxy, produce a stable stationary waterproof supercapacitor module. Our supercapacitor technology adds value to a "dirt-cheap" construction material and demonstrates a scalable process affording energy storage for powering embedded microdevices in architectural applications that utilize fired brick.

\section{Methods}

Materials. Chlorobenzene (99\%), 3,4-ethylenedioxythiophene (97\%), poly(vinyl alcohol) $\left(M_{\mathrm{w}} 89,000-98,000,99+\%\right.$ hydrolyzed), methanol ( $\left.\geq 99.8 \%\right)$, and hydrochloric acid (37\%) are purchased from Sigma-Aldrich; sulfuric acid (AR) is purchased from Macron. The PEDOT:PSS solution (Clevios PH 1000) is purchased from Heraeus company. All chemicals are used without further purification. Platinum foil $(0.025 \mathrm{~mm}$ thick, $99.9 \%)$ is purchased from Alfa Aesar and utilized for engineering electrode leads and Celgard 3501 membrane is used as a separator. Fired bricks are purchased from local hardware stores: The Home Depot Inc. (type 1 brick, https://www.homedepot.com, Internet \#100323015, Model \#RED0126MCO, Store SKU \#393134), Lowe's Inc. (type 2 brick, https://www.lowes.com, Item \# 10298, Model \# 600370) and Menards Inc. (type 3 brick, https://www.menards.com, Model
Number: 1441901, Menards ${ }^{\circledast}$ SKU: 1441901). Road pavers are purchased from Menards Inc. (https://www.menards.com, Model Number: 1793028, Menards ${ }^{\circledast}$ SKU: 1793028) and all construction materials used for developing electrodes are cut using a diamond saw. Red cement color $\left(\alpha-\mathrm{Fe}_{2} \mathrm{O}_{3}\right.$ particles) produced by NewLook Inc. is purchased from The Home Depot Inc. (https://www.homedepot.com, Internet \#203858654, Model \#CC1LB105) and serves as an oxidant source for developing chemical syntheses. Materials for making concrete (purchased from The Home Depot Inc., https://www.homedepot.com) include commercial-grade Quikrete Portland cement (Type I/II) (Internet \#100318486, Model \#100700, Store SKU \#616788), Quikrete all-purpose sand (Internet \#100318450Model \# 115251Store SKU \#137263) and Pavestone multi-purpose patio/paver base (Internet \#100580973, Model \#98001, Store SKU \#208618).

Characterization. Scanning electron micrographs and energy-dispersive X-ray spectra are collected with a JEOL 7001LVF FE-SEM. Two-point probe resistance measurements are carried out using a Fluke 177 True RMS digital multimeter with $3 \mathrm{~mm}$ distance between two probes. Thermogravimetric analysis is conducted on a Discovery TGA (TA Instruments). Cyclic voltammetry, galvanostatic

charge-discharge measurements and electrochemical impedance spectroscopy are performed in a BioLogic VMP3 multipotentiostat. For electrochemical impedance spectroscopy, the sinusoidal disturbance is $10 \mathrm{mV}$ with frequencies scanned between $100 \mathrm{kHz}$ and $0.1 \mathrm{~Hz}$. A Nyquist plot shows real impedance $Z^{\prime}$ vs. imaginary impedance $-Z^{\prime \prime}$ under a sinusoidal disturbance at the open circuit potential. Fitting of Nyquist plot using an equivalent circuit diagram contains solution resistance $\left(R_{\mathrm{s}}\right)$, electrode material resistance $\left(R_{\mathrm{m}}\right)$, material capacitance $\left(C_{\mathrm{m}}\right)$, double-layer capacitance $\left(C_{\mathrm{dl}}\right)$, and constant phase element $(\mathrm{CPE})$. Here, $R_{\mathrm{s}}$ reflects the electrolyte ionic mobility and $R_{\mathrm{m}}$ represents the electrical resistance of the electrode. Powder X-ray diffraction spectra of brick powders pulverized by mortar and pestle are obtained in a Bruker $\mathrm{d} 8$ Advance X-ray diffractometer at room temperature, with a Cu Ka radiation source $(\lambda=1.5406 \AA)$ and LynxEyeXe detector. The sample holder is a background-free silicon disk rotating at $30 \mathrm{rpm}$ when collecting data with a $0.02^{\circ}$ scan step at $40 \mathrm{kV}$ and $40 \mathrm{~mA}$. Current-voltage tests are performed on a $3 \mathrm{D}$ printed two-point probe station with two gold probes separated by $2 \mathrm{~mm}^{23}$. Water absorption experiments are performed as described in ASTM C67/C67M-18 except brick samples are $1 \mathrm{~cm} \times 0.5 \mathrm{~cm} \times 0.28 \mathrm{~cm}$ in size. Inductively coupled plasma mass spectrometry is performed on a Perkin Elmer 
ELAN DRC II ICP-MS. Samples for testing are obtained from electrolytes $(5 \mathrm{~mL}$ $1 \mathrm{M} \mathrm{H}_{2} \mathrm{SO}_{4}$ ) after three-electrode cyclic voltammetry experiments and are diluted to $1 / 100$ with Mili-Q water before analyses. External calibration curves are obtained with IV-ICPMS-71A standard solution purchased from Inorganic Ventures, Inc.

Preparation of poly(vinyl alcohol) $/ \mathbf{H}_{\mathbf{2}} \mathbf{S O}_{\mathbf{4}}$ gel electrolyte. The gel electrolyte is formulated using $1 \mathrm{~g}$ of poly(vinyl alcohol) powder dissolved in $10 \mathrm{~mL}$ deionized water under vigorous stirring at $90^{\circ} \mathrm{C}$ and cooled to around $50{ }^{\circ} \mathrm{C}$. Dropwise addition of $1 \mathrm{~g}$ of concentrated $\mathrm{H}_{2} \mathrm{SO}_{4}(1 \mathrm{M})$ is then carried out by pipetting acid on the inner wall under vigorous stirring to prevent carbonization of poly(vinyl alcohol). Stirring minimizes localized heating and is carried out for $1 \mathrm{~h}$ resulting in a homogeneous, translucent, and colorless solution.

Synthesis of nanofibrillar PEDOT coating on a brick. Brick is cut using a diamond saw $( \pm 0.03 \mathrm{~cm}$ error) into the following 4 different sizes: $1.00 \mathrm{~cm} \times 0.50 \mathrm{~cm} \times$ $0.28 \mathrm{~cm}$ (for studying synthesis and electrochemistry), $1.27 \mathrm{~cm} \times 1.27 \mathrm{~cm} \times 0.20 \mathrm{~cm}$ (for patterning), $2.00 \mathrm{~cm} \times 1.00 \mathrm{~cm} \times 1.00 \mathrm{~cm}$ (for scaled-up supercapacitor) and $10.16 \mathrm{~cm} \times 6.77 \mathrm{~cm} \times 5.72 \mathrm{~cm}$ (for scaled-up synthesis). A brick is thrice washed with deionized water to remove surface dust then dried at $160^{\circ} \mathrm{C}$ for $1 \mathrm{~h}$ and cooled to room temperature.

The syntheses of all types of $1 \mathrm{~cm} \times 0.5 \mathrm{~cm} \times 0.28 \mathrm{~cm}$ brick are performed in a $25 \mathrm{~mL}$ Teflon-lined stainless-steel autoclave as shown in Fig. 1a. A brick is placed on a glass reservoir then $200 \mu \mathrm{L}$ of a $0.85 \mathrm{M} \mathrm{EDOT/chlorobenzene} \mathrm{solution} \mathrm{is}$ loaded in a separate glass reservoir and $30 \mu \mathrm{L}$ of $12 \mathrm{M} \mathrm{HCl}$ is directly injected in the Teflon liner. The reactor is closed and introduced into an oven at $160^{\circ} \mathrm{C}$ for $14 \mathrm{~h}$. The product is washed thrice with excess methanol and dried at room temperature before carrying out tests. Similarly, the synthesis of a $2 \mathrm{~cm} \times 1 \mathrm{~cm} \times 1 \mathrm{~cm}$ brick is performed in a $125 \mathrm{~mL}$ Teflon-lined stainless-steel autoclave using $600 \mu \mathrm{L}$ of a $0.70 \mathrm{M}$ EDOT in chlorobenzene solution and $75 \mu \mathrm{L}$ of $12 \mathrm{M} \mathrm{HCl}$.

A brick $(1.27 \mathrm{~cm} \times 1.27 \mathrm{~cm} \times 0.20 \mathrm{~cm})$ is patterned using a polyimide tape mask. Synthesis is carried out at $150^{\circ} \mathrm{C}$ for $14 \mathrm{~h}$ in a $125 \mathrm{~mL}$ Teflon-lined stainless-steel autoclave containing $1 \mathrm{~mL}$ of a $0.85 \mathrm{M}$ EDOT solution in chlorobenzene and $0.6 \mathrm{~mL}$ of $12 \mathrm{M} \mathrm{HCl}$ (Supplementary Fig. 4a).

Scaling is carried in a glass reactor $(12.30 \mathrm{~cm} \times 8.55 \mathrm{~cm} \times 11.30 \mathrm{~cm})$ with a large brick $(10.16 \mathrm{~cm} \times 6.77 \mathrm{~cm} \times 5.72 \mathrm{~cm})$. The reaction is carried out using $15 \mathrm{~mL}$ of $12 \mathrm{M} \mathrm{HCl}$ and $15 \mathrm{~mL}$ of a $0.85 \mathrm{M} \mathrm{EDOT}$ in chlorobenzene solution at $150^{\circ} \mathrm{C}$ for $6 \mathrm{~h}$ (Fig. 2e).

We produce a PEDOT coating on concrete by applying $\alpha-\mathrm{Fe}_{2} \mathrm{O}_{3}$ particles to a concrete surface (Supplementary Fig. 4c). This composite is produced by mixing sand, stone, Portland cement and water in a weight ratio of 3:1.5:1:0.7. An uncured concrete slurry is then injected into a $1.27 \mathrm{~cm} \times 1.27 \mathrm{~cm} \times 2.54 \mathrm{~cm}$ mold, stirred to remove gas bubbles, and cured for 3 days in ambient conditions. A partially cured concrete bar is dipped in an aqueous dispersion of $\alpha-\mathrm{Fe}_{2} \mathrm{O}_{3}\left(0.25 \mathrm{~g} \mathrm{~mL}^{-1}\right)$ for $3 \mathrm{~s}$, then dried in air. Synthesis is performed at $150^{\circ} \mathrm{C}$ for $14 \mathrm{~h}$ in a $125 \mathrm{~mL}$ Teflon-lined stainless-steel autoclave loaded with $1 \mathrm{~mL}$ of a $0.45 \mathrm{M}$ EDOT in chlorobenzene solution and $0.1 \mathrm{~mL}$ of $12 \mathrm{M} \mathrm{HCl}$.

Fabrication of a quasi-solid-state supercapacitor. The brick is attached to Pt as shown in Supplementary Fig. 7a (left top) prior to gel casting. The gel electrolyte $\left(0.1 \mathrm{~g} \mathrm{~mL}^{-1}\right.$ poly (vinyl alcohol) $\left./ 1 \mathrm{M} \mathrm{H}_{2} \mathrm{SO}_{4}\right)$ is pipetted onto two $1 \mathrm{~cm} \times 0.5 \mathrm{~cm} \times$ $0.28 \mathrm{~cm}$ PEDOT-coated bricks ( $100 \mu \mathrm{L}$ each brick on the $1 \mathrm{~cm} \times 0.5 \mathrm{~cm}$ face). This electrolyte is allowed to impregnate for $12 \mathrm{~h}$ at ambient conditions $\left(25^{\circ} \mathrm{C}, 30-60 \%\right.$ relative humidity) forming a semidry gel layer. An additional $25 \mu \mathrm{L}$ of gel electrolyte is added serving as a binder between two bricks to assemble a sandwich-type supercapacitor. A device is dried in ambient conditions $\left(25^{\circ} \mathrm{C}, 30-60 \%\right.$ relative humidity) for $1 \mathrm{~h}$ before sealing with epoxy (Supplementary Fig. 9a).

Fabrication of a tandem supercapacitor. The gel electrolyte is a mixture of $0.1 \mathrm{~g}$ of poly(vinyl alcohol) in $1 \mathrm{~mL}$ of $1 \mathrm{M} \mathrm{H}_{2} \mathrm{SO}_{4}$ and $200 \mu \mathrm{L}$ are added to a PEDOTcoated brick face $(1 \mathrm{~cm} \times 1 \mathrm{~cm})$. This electrolyte is allowed to stabilize for $12 \mathrm{~h}$ at ambient conditions $\left(25^{\circ} \mathrm{C}, 30-60 \%\right.$ relative humidity); this process is repeated for all brick electrodes resulting in a thick gel layer (Supplementary Fig. 11a). An additional $50 \mu \mathrm{L}$ of poly(vinyl alcohol) $/ \mathrm{H}_{2} \mathrm{SO}_{4}$ solution is added serving as a binder between bricks. Devices are dried in an oven at $50^{\circ} \mathrm{C}$ for $2 \mathrm{~h}$ and platinum foil, serving as a lead, is attached to bricks using polyimide tape.

\section{Data availability}

The data that support the findings of this study are available from the corresponding author on request.

Received: 21 December 2019; Accepted: 16 June 2020; Published online: 11 August 2020

\section{References}

1. Yang, Y., Yu, S.-Y., Zhu, Y. \& Shao, J. The making of fired clay bricks in China some 5000 years ago. Archaeometry 56, 220-227 (2014).
2. Kreimeyer, R. Some notes on the firing colour of clay bricks. Appl. Clay Sci. 2, 175-183 (1987).

3. Henshilwood, C. S. et al. An abstract drawing from the 73,000-year-old levels at Blombos Cave, South Africa. Nature 562, 115-118 (2018).

4. Proceedings of the National Academy of Sciences Roebroeks W. et al. Use of red ochre by early Neandertals. Proc. Natl Acad. Sci. USA 109, 1889-1894 (2012).

5. Liu, Q. et al. Aligned Fe2TiO5-containing nanotube arrays with low onset potential for visible-light water oxidation. Nat. Commun. 5, 5122 (2014).

6. Jia, C.-J. et al. Large-scale synthesis of single-crystalline iron oxide magnetic nanorings. J. Am. Chem. Soc. 130, 16968-16977 (2008).

7. Kenel, C., Casati, N. P. M. \& Dunand, D. C. 3D ink-extrusion additive manufacturing of $\mathrm{CoCrFeNi}$ high-entropy alloy micro-lattices. Nat. Commun. 10, 904 (2019).

8. Yang, F. et al. Yolk-shell structured FeP@C nanoboxes as advanced anode materials for rechargeable lithium-/potassium-ion batteries. Adv. Funct. Mater. 29, 1808291 (2019).

9. Han, S., Hu, X., Wang, J., Fang, X. \& Zhu, Y. Novel route to Fe-based cathode as an efficient bifunctional catalysts for rechargeable Zn-Air battery. Adv. Energy Mater. 8, 1800955 (2018).

10. Dong, Y. et al. Air-stable porous Fe2N encapsulated in carbon microboxes with high volumetric lithium storage capacity and a long cycle life. Nano Lett. 17, 5740-5746 (2017).

11. Zhan, C. et al. Enabling the high capacity of lithium-rich anti-fluorite lithium iron oxide by simultaneous anionic and cationic redox. Nat. Energy 2, 963-971 (2017).

12. Owusu, K. A. et al. Low-crystalline iron oxide hydroxide nanoparticle anode for high-performance supercapacitors. Nat. Commun. 8, 14264 (2017).

13. Diao, Y. et al. Converting rust to PEDOT nanofibers for supercapacitors. ACS Appl. Energy Mater. 2, 3435-3444 (2019).

14. Wang, X. et al. High electrical conductivity and carrier mobility in oCVD PEDOT thin films by engineered crystallization and acid treatment. Sci. Adv. 4, eaat5780 (2018).

15. Berggren, M. \& Malliaras, G. G. How conducting polymer electrodes operate Science 364, 233-234 (2019).

16. Zhang, S. et al. p-Toluenesulfonic acid catalytic polymerization of EDOT without oxidants. Mater. Lett. 222, 105-108 (2018).

17. Xiao, H. et al. Stretchable tandem micro-supercapacitors with high voltage output and exceptional mechanical robustness. Energy Storage Mater. 13, 233-240 (2018).

18. Wang, H. et al. Self-woven nanofibrillar PEDOT mats for impact-resistant supercapacitors. Sustain. Energy Fuels 3, 1154-1162 (2019).

19. Ding, M. et al. Effect of $\mathrm{Fe}(\mathrm{III})$ on the positive electrolyte for vanadium redox flow battery. R. Soc. Open Sci. 6, 181309 (2019).

20. Li, X. et al. High energy flexible supercapacitors formed via bottom-up infilling of gel electrolytes into thick porous electrodes. Nat. Commun. 9, 2578 (2018).

21. Zhou, H. et al. Printable fabrication of Pt-and-ITO free counter electrodes for completely flexible quasi-solid dye-sensitized solar cells. J. Mater. Chem. A 1, 3932-3937 (2013).

22. Dubal, D. P., Chodankar, N. R., Kim, D. H. \& Gomez-Romero, P. Towards flexible solid-state supercapacitors for smart and wearable electronics. Chem Soc. Rev. 47, 2065-2129 (2018).

23. Lu, Y., Santino, L. M., Acharya, S., Anandarajah, H. \& D'Arcy, J. M. Studying electrical conductivity using a 3D printed four-point probe station. J. Chem. Educ. 94, 950-955 (2017).

\section{Acknowledgements}

We thank Dr. Bryce Sadtler from the Department of Chemistry and Dr. Anne M. Hofmeister from the Department of Earth and Planetary Sciences at Washington University for providing fruitful discussions. We are grateful to Dr. Huafang Li from the Institute of Materials Science \& Engineering at Washington University for helping with electron microscopy. We also acknowledge helpful technical input from Dr. Luciano M. Santino and Mr. Haozhe Chen and are grateful to Dr. G.S. D'Arcy for editing this work

\section{Author contributions}

H.W. and J.M.D. designed the experiments. H.W. carried out the synthesis, device fabrication and characterization. Q.Z. performed inductively coupled plasma mass spectrometry characterization and analyses. H.W. and J.M.D. analyzed data and wrote the paper. H.W., Y.D., Y.L., H.Y., Q.Z., K.C. and J.M.D. contributed to the discussion and editing of the paper.

\section{Competing interests}

The authors declare no competing interests. 


\section{Additional information}

Supplementary information is available for this paper at https://doi.org/10.1038/s41467020-17708-1.

Correspondence and requests for materials should be addressed to J.M.D.

Peer review information Nature Communications thanks Yu Wang and the other, anonymous, reviewer(s) for their contribution to the peer review of this work. Peer reviewer reports are available.

Reprints and permission information is available at http://www.nature.com/reprints

Publisher's note Springer Nature remains neutral with regard to jurisdictional claims in published maps and institutional affiliations. (c) (i) Open Access This article is licensed under a Creative Commons Attribution 4.0 International License, which permits use, sharing, adaptation, distribution and reproduction in any medium or format, as long as you give appropriate credit to the original author(s) and the source, provide a link to the Creative Commons license, and indicate if changes were made. The images or other third party material in this article are included in the article's Creative Commons license, unless indicated otherwise in a credit line to the material. If material is not included in the article's Creative Commons license and your intended use is not permitted by statutory regulation or exceeds the permitted use, you will need to obtain permission directly from the copyright holder. To view a copy of this license, visit http://creativecommons.org/ licenses/by/4.0/.

(C) The Author(s) 2020 